Article

\title{
Comparison of Sun-Induced Chlorophyll Fluorescence Estimates Obtained from Four Portable Field Spectroradiometers
}

\author{
Tommaso Julitta ${ }^{1, *}$, Lawrence A. Corp ${ }^{2}$, Micol Rossini ${ }^{1}$, Andreas Burkart ${ }^{3}$, Sergio Cogliati ${ }^{1}$, \\ Neville Davies ${ }^{4}$, Milton Hom ${ }^{2}$, Alasdair Mac Arthur ${ }^{5}$, Elizabeth M. Middleton ${ }^{6}$, Uwe Rascher ${ }^{3}$, \\ Anke Schickling ${ }^{3}$ and Roberto Colombo ${ }^{1}$ \\ 1 Remote Sensing of Environmental Dynamics Lab., DISAT, Università di Milano-Bicocca, \\ P.zza della Scienza 1, 20126 Milan, Italy; micol.rossini@unimib.it (M.R.); sergio.cogliati@unimib.it (S.C.); \\ roberto.colombo@unimib.it (R.C.) \\ 2 Science Systems and Applications Inc., Lanham, MD 20706, USA; lawrence.a.corp@nasa.gov (L.A.C.); \\ milton.g.hom@nasa.gov (M.H.) \\ 3 Institute of Bio- and Geosciences, IBG-2: Plant Sciences, Forschungszentrum Jülich GmbH, 52425 Jülich, \\ Germany; an.burkart@fz-juelich.de (A.B.); u.rascher@fz-juelich.de (U.R.); a.schickling@fz-juelich.de (A.S.) \\ 4 Ocean Optics, Oxford OX4 2JY, UK; neville.davies@oceanoptics.eu \\ 5 NERC Field Spectroscopy Facility, School of Geosciences, University of Edinburgh, Grant Institute, \\ Kings Buildings, West Mains Road, Edinburgh EH9 3FE, UK; alasdair.macarthur@ed.ac.uk \\ 6 Biospheric Sciences Laboratory, NASA/Goddard Space Flight Center, Greenbelt, MD 20771, USA; \\ elizabeth.m.middleton@nasa.gov \\ * Correspondence: tommaso.julitta@unimib.it; Tel.: +39-026-448-2848; Fax: +39-026-448-2895
}

Academic Editors: Eyal Ben-Dor, Clement Atzberger and Prasad S. Thenkabail

Received: 25 September 2015; Accepted: 29 January 2016; Published: 5 February 2016

\begin{abstract}
Remote Sensing of Sun-Induced Chlorophyll Fluorescence (SIF) is a research field of growing interest because it offers the potential to quantify actual photosynthesis and to monitor plant status. New satellite missions from the European Space Agency, such as the Earth Explorer 8 FLuorescence EXplorer (FLEX) mission—scheduled to launch in 2022 and aiming at SIF mapping - and from the National Aeronautics and Space Administration (NASA) such as the Orbiting Carbon Observatory-2 (OCO-2) sampling mission launched in July 2014, provide the capability to estimate SIF from space. The detection of the SIF signal from airborne and satellite platform is difficult and reliable ground level data are needed for calibration/validation. Several commercially available spectroradiometers are currently used to retrieve SIF in the field. This study presents a comparison exercise for evaluating the capability of four spectroradiometers to retrieve SIF. The results show that an accurate far-red SIF estimation can be achieved using spectroradiometers with an ultrafine resolution (less than $1 \mathrm{~nm}$ ), while the red SIF estimation requires even higher spectral resolution (less than $0.5 \mathrm{~nm}$ ). Moreover, it is shown that the Signal to Noise Ratio (SNR) plays a significant role in the precision of the far-red SIF measurements.
\end{abstract}

Keywords: sun-induced chlorophyll fluorescence (SIF); field spectroscopy; sensor characteristics; SIF retrieval methods; Fraunhofer line depth (FLD); spectral resolution; signal to noise ratio (SNR); SIF calibration/validation

\section{Introduction}

For decades, active chlorophyll-a fluorescence measurements have been used, both in laboratory and in the field, to better describe physiological processes of plants, such as photosynthesis, carbon fixation and stress [1]. More recently, techniques for the measurement of the top of canopy Sun-Induced 
Fluorescence (SIF) from remote sensing platforms have been developed, opening unprecedented opportunity to monitor canopy fluorescence at the ecosystem scale. This will lead to a better understanding of the significance of spatial variability in the SIF signal and will facilitate the up-scaling from the canopy to the landscape levels [2].

SIF is an electromagnetic signal emitted throughout the red and near-infrared (NIR) spectrum by chlorophyll-a, the primary photosynthetic pigment in green vegetation, in response to the absorption of photosynthetically active radiation from the sun. This low signal, typically $1 \%-5 \%$ of the reflected radiation in the NIR, is emitted by the photosynthetic apparatus. Over the past two decades, research has demonstrated the potential use of SIF to monitor photosynthesis and the functional status of vegetation [3-5]. In recent years, the growing interest of the scientific community in remote sensing of SIF is attested by the increasing number of scientific studies based on SIF estimation at different scales of investigation [6-8]. The global estimation of SIF from space is particularly relevant due to its potential for improving our ability to accurately quantify terrestrial photosynthesis and to monitor plant functional status of ecosystems. This is the scientific rational behind the development of the FLuorescence EXplorer (FLEX), a European Space Agency (ESA) Earth Explorer 8 Mission (a report the mission at the following link: http:/ / esamultimedia.esa.int/docs/EarthObservation/SP1330-2_FLEX.pdf). FLEX, currently scheduled for a 2022 launch, will obtain full spectrum red and far-red SIF emissions [9], as well as other biophysical properties using traditional visible-NIR spectral reflectance indices provided by its tandem orbit with the ESA SENTINEL-3 mission. At the same time, the newly launched NASA Orbiting Carbon Observatory-2 (OCO-2) has been considered a suitable sensor for retrieving samples of the far-red SIF signal at orbital altitudes.

The SIF signal is small relative to reflected radiation, so its detection from airborne and satellite platforms is difficult, and an accurate atmospheric correction is needed. At ground level, the atmospheric influence in the reflected radiance is negligible. In this context, field spectroscopy is considered as a valuable tool for evaluating technical requirements for SIF retrieval. Furthermore, ground level estimates of SIF are useful for improving our understanding of the relationships between SIF and additional biophysical properties of vegetation and to aid in the calibration/validation of airborne- and satellite-derived SIF data. The challenge for SIF retrievals lies in the difficulty to decouple the weak emitted SIF signal from the dominating reflected radiance. The estimation of SIF from radiances recorded both at top of canopy (TOC) [10-12] or top of atmosphere [13-18] exploits regions of the atmospheric spectrum where the incident irradiance is strongly reduced due to absorption in the Earth's atmosphere or in the solar atmosphere. Two telluric oxygen absorption features are frequently exploited for SIF retrievals namely, $\mathrm{O}_{2}-\mathrm{B}$ and $\mathrm{O}_{2}-\mathrm{A}$ bands centered at $687.0 \mathrm{~nm}$ and $760.4 \mathrm{~nm}$, respectively. The chlorophyll fluorescence emission spectrum is characterized by two peaks in the red and in the far-red regions, approximately centered at $690 \mathrm{~nm}$ and $740 \mathrm{~nm}$, respectively [19]. The $\mathrm{O}_{2}-\mathrm{B}$ band is used to retrieve red SIF (at $687 \mathrm{~nm}$ ); the $\mathrm{O}_{2}-\mathrm{A}$ band can be used for the far-red SIF estimates (at $760 \mathrm{~nm}$ ). To detect these narrow atmospheric absorption bands, instruments having fine (Full Width at Half Maximum (FWHM) of $1-5 \mathrm{~nm}$ ) or ultrafine (FWHM $<1 \mathrm{~nm}$ ) spectral resolution is required, and different methods have been proposed for SIF retrieval. The ability to apply one method or another often relies on the spectral resolution and on the dynamic range of the device used. The Spectral Fitting Methods proposed by Meroni et al. [20] and the statistical approaches [11] require spectra collected with ultrafine resolution, while the Fraunhofer Line Discriminator (FLD) method [21] or its several different formulations (single FLD (sFLD)), three FLD (3FLD) or improved FLD (iFLD) (see Meroni et al. [6] for a review of algorithms) can be applied on fine resolution spectra [22].

Despite the fact that several research groups are now proposing field prototypes based on different spectroradiometer models to monitor TOC SIF [10,23-27], no studies have been performed comparing SIF retrievals from different systems. In the context of calibration/validation activities, the definition of the proper set-up for reliable SIF estimates is needed. This requires a network of stable sensors that follow the same measurement standards and calibration protocols to allow across-site 
comparisons [28]. To the best of our knowledge, only one study [22] quantified the expected impacts of sensor characteristics, such as spectral resolution (SR), spectral sampling interval (SSI), stability, and signal to noise ratio (SNR) on the accuracy of SIF retrieval. However, that study only examined the potential retrieval of SIF in the far-red $\left(\mathrm{O}_{2}-\mathrm{A}\right.$ band $)$ region and was exclusively based on modeled data.

The present study provides quantitative assessment of the reproducibility of SIF retrieval at both oxygen bands using radiance spectra simultaneously acquired from four spectroradiometers. These include three miniaturized systems from Ocean Optics; two HR4000 and one QE Pro (http://www.oceanoptics.com/) and one ASD Fieldspec Pro (http://www.asdi.com/). The impact of the different device characteristics (e.g., SR, SSI and SNR) on the SIF estimates has been analyzed and discussed and indications on the best performing instruments are provided. We want to clearly state that we are not making any commercial product recommendations. In the analysis, we considered a limited number of instruments currently in use, operated following the most common protocols used in the field to estimate SIF. The selected spectroradiometers are assumed to be representative of the model tested. Further in depth comparisons should be performed (e.g., including other instruments not considered in this comparison and multiple instruments of each type) and we recognize that improvements in the performances of all of the tested instruments might be achieved with optimal settings determined through laboratory analysis. This study therefore offers a preliminary cross examination of commercial off the shelf components that are currently being deployed in prototype tower based SIF observing stations, such as University of Milano Bicocca's Multiplexer Radiometer Irradiometer (Milan, Italy), Jülich Research Center's S-FLUO Box (Jülich, Germany), NASA Goddard's FUSION (Greenbelt, MA, USA), and the CNRS TriFLEX (Paris, France), could be used in ground validation stations to support the FLEX Earth Explorer 8 Mission.

\section{Methods}

Four spectroradiometers with different characteristics in terms of SR, SSI, and SNR have been considered. The devices were used to collect measurements simultaneously above the same vegetated target (a $50 \mathrm{~cm} \times 40 \mathrm{~cm}$ square area of lawn grass, Festuca arundinacea). The same measurement protocol, data analysis procedure and algorithm for SIF estimation were used for all evaluations. Leaf level fluorescence emissions were also measured and used for comparison with the SIF values estimated at canopy level. Indeed, top of canopy SIF estimates cannot be validated except with contemporary in situ measurements exploiting radiative transfer modeling techniques or through comparison with a fluorescence reference with known intensity in the red and far-red region [29].

The spectroradiometers used in this study were: Ocean Optics HR4000 narrow range (spectral range: $670-857 \mathrm{~nm}$ ), Ocean Optics QE Pro (spectral range: 645-810 nm), Ocean Optics HR4000 full range (spectral range: 197-1115 nm) and ASD FieldSpec Pro (spectral range: 350-2500 nm), hereafter called HRNR, QE, HRFR and ASD, respectively. The instrument characteristics are summarized in Table 1, and the description of the methods used to characterize the devices is reported below in Section 2.1. Two HR4000s (HRNR and HRFR) were hosted in the S-FLUO box, a prototype system owned by the Jülich Research Center (Jülich, Germany), and designed for high temporal frequency acquisition of continuous radiometric measurements. The S-FLUO box is based on a commercial optical multiplexer (MPM-2000, Ocean Optics, Dunedin, FL, USA), able to switch between a channel measuring the incident irradiance (cosine response optic), a down-looking bare fiber ( $25^{\circ}$ of Field Of View, FOV) for the measurement of the upwelling radiance and a blind channel for the spectral dark current measurement [27]. A thermo-regulated system holds the temperature constant at $25^{\circ} \mathrm{C}$ while QE is automatically thermo-regulated and the ASD has been operated as commonly used in the field, at ambient temperature.

\subsection{Instrument Characterizations}

All the instruments have been characterized in terms of radiometric response, spectral noise and resolution in order to properly compare the results. An additional element considered during the 
analysis has been the oxygen absorption band depth. The analysis was conducted in the laboratory and outdoors, before the intercomparison experiment.

\subsubsection{Signal to Noise Ratio and Full Width at Half Maximum Characterization}

The SNR characterization was accomplished following the protocol defined by Schaepman and Dangel [30]. The SNR of a spectroradiometer varies within the spectrum and it has been expressed here as average value within the spectral range covered by each instrument: $670-857 \mathrm{~nm}$ for HRNR, 645-810 nm for QE, 197-1115 for HRFR and 350-2500 for ASD. The mean SNR values are reported in Table 1.

The spectral resolution, expressed as full width at half maximum (FWHM), of each device was determined by exploiting the SpecCal tool [31,32], which is based on the comparison between measured and modeled solar irradiance spectra. According to the spectral range of each device the number of atmospheric absorption features used for the FWHM determination varies between 4 (for the HRNR) and 11 (for the ASD). The mean FWHM values are reported in Table 1.

\subsubsection{Oxygen Absorption Band Depth}

The ability of the spectroradiometers to resolve the $\mathrm{O}_{2}$ absorption bands is related to their spectral resolution, their spectral sampling interval, and to a lesser extent, their dynamic range, with the ability to discern increasing feature depth aided by higher spectral resolution. For this reason, the $\mathrm{O}_{2}$ absorption band depth can be considered an indicator of the capability of each device to detect the absorption features within the $\mathrm{O}_{2}-\mathrm{B}(687.0 \mathrm{~nm})$ and $\mathrm{O}_{2}-\mathrm{A}(760.4 \mathrm{~nm})$ bands, respectively, even if the depths vary somewhat due to the sun's position and/or the direct/diffuse shortwave radiation.

The absorption band depths have been calculated as follows:

$$
\text { Band depth }=\frac{L^{\downarrow} \lambda \text { out }-L^{\downarrow} \lambda \text { in }}{L^{\downarrow} \lambda \text { out }} \times 100
$$

where $\mathrm{L}^{\downarrow}$ is the incident solar radiance. $\lambda$ in refers to the wavelength at the bottom of the $\mathrm{O}_{2}$ feature, around $689 \mathrm{~nm}$ for the $\mathrm{O}_{2}-\mathrm{B}$ band or $760 \mathrm{~nm}$ for the $\mathrm{O}_{2}-\mathrm{A}$ band. $\lambda$ out refers to the wavelength at the shoulder that provides the maximum value in the range of $680-688 \mathrm{~nm}$ for the $\mathrm{O}_{2}-\mathrm{B}$ band or $750-755$ $\mathrm{nm}$ for the $\mathrm{O}_{2}$-A band. Measurements at these wavelengths were used to determine the feature depth values associated with each spectrometer and are reported in Table 1.

\subsection{Experiment Setup and Canopy Fluorescence Measurements}

The experiment was conducted at the NASA Goddard Space Flight Center in Greenbelt, Maryland USA ( $\left.38^{\circ} 59^{\prime} 32.15^{\prime \prime} \mathrm{N}, 76^{\circ} 50^{\prime} 22.22^{\prime \prime} \mathrm{W}\right)$. The measurements were acquired between 11:00 a.m. and 1:00 p.m. solar time (Solar Zenith Angles, 27-29 degrees) on 24 April 2014 under clear sky conditions. Each measurement cycle consisted of four spectra: first, a measurement of the dark current followed by 3 spectra whereby a measurement of the target's up-welling radiance was sandwiched between two solar irradiance measurements (e.g., dark current, irradiance, target, and irradiance). The solar irradiance at the time of the target measurement was estimated by linear interpolation over the cycle's 1 minute duration [33].

For the HR4000s (HRNR, HRFR), the two sensors hosted in the S-FLUO box were used to measure the up-welling target radiance (field of view, FOV of 25 degrees) with the downward channels and the solar irradiance with the upward channels (Ocean Optics cosine receptors were used). The S-FLUO box's downward channels were kept fixed above the same grass area using a second tripod, while the upward channels were secured to a third leveled tripod looking at the sky. The QE and the ASD Fieldspec spectroradiometers were operated with bare fiber optics where the FOV was 25 and 18 degree's respectively. A rotating tripod arm with a fiber optic holder was used to ensure that the fibers of the QE, HRNR, HRFR and ASD were as close together as possible in order to measure as 
similar a spatial area on the target as could be physically achieved (Figure 1). The rotating arm was facing south, avoiding any possible shadow both on the white reference panel and on the vegetation target. The fiber optics of QE and ASD acquired alternating measurements of a calibrated white spectralon panel (99\% reflecting Labsphere Inc., North Sutton, NH, USA) and the up-welling radiance of the grass.

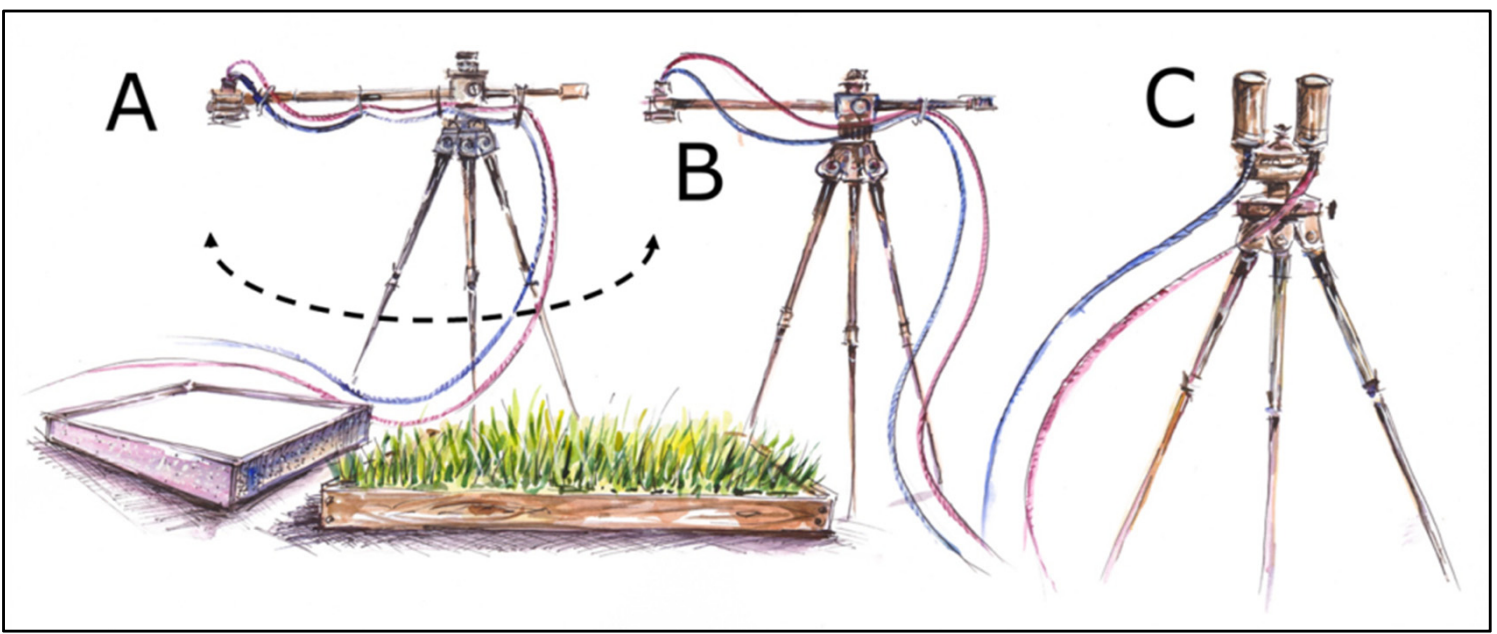

Figure 1. Experimental field setup: (A) The fiber optics connected to ASD Filedspec Pro and QE Pro spectroradiometers are secured to a rotating arm allowing the alternating movement from the white reference panel and the vegetation target; (B) The S-FLUO box downward channels (both HR4000 Narrow Range and HR4000 Full Range) are attached to a fixed tripod looking at the vegetation; (C) S-FLUO box upward channels are horizontally mounted on the third tripod to collect reference measurements.

The grass target was observed from nadir at an average distance of $30 \mathrm{~cm}$ corresponding to a viewed area of approximately 10 or $15 \mathrm{~cm}$ diameter for all spectroradiometers. The integration time of the sensors was set to optimize the signal at around $80 \%$ of the dynamic range of each device. Spectrum averages varied according to the instrument, the selection has been made in order to maximize the number of scan repetition without exceeding 3 minutes per each cycle. In particular, the number of spectra averaged was set to: 3 for HRNR, 5 for HRFR, 5 for QE and 25 for ASD. The instruments were warmed up for $1.5 \mathrm{~h}$ before beginning the data acquisition. A total of 30 cycles of measurements were collected at the same time by each of these different instruments.

\subsection{SIF Retrieval}

The different spectral resolution of the spectroradiometers did not allow us to perform any kind of algorithm comparison. Therefore, the SIF retrievals were conducted with FLD-like approaches that were applicable to all of the spectroradiometers used in this study. The 3FLD method was used to estimate far-red SIF for the $\mathrm{O}_{2}-\mathrm{A}$ band, considered to be the best option following Damm et al. [22]. The assumption of the 3FLD method cannot be considered valid for the red SIF retrieval at the $\mathrm{O}_{2}$-B-band. Therefore, the sFLD method has been used to retrieve SIF at the $\mathrm{O}_{2}$-B-band, using the following formula:

$$
\mathrm{F}=\frac{L^{\downarrow} \lambda \text { out } \times L^{\uparrow} \lambda \text { in }-L^{\uparrow} \lambda \text { out } \times L^{\downarrow} \lambda \text { in }}{L^{\downarrow} \lambda \text { out }-L^{\downarrow} \lambda \text { in }}
$$


where $L^{\downarrow}$ is the incident solar radiance and $L^{\uparrow}$ is the target radiance. $\lambda$ in and $\lambda$ out refer to the wavelengths at the bottoms and at the shoulders of the absorption features, respectively. To minimize possible mismatches due to the wavelength calibrations, an automatic selection of local maxima (outside of the absorption band) and minima (inside the absorption band) was used. In particular, the radiances associated with the deepest absorption band positions at $689 \mathrm{~nm}$ and $760 \mathrm{~nm}$ were selected to determine $\mathrm{L}^{\downarrow} \lambda$ in and $\mathrm{L}^{\uparrow} \lambda$ in for the $\mathrm{O}_{2}-\mathrm{B}$ and $\mathrm{O}_{2}$-A bands, respectively. $\mathrm{L}^{\uparrow} \lambda$ out and $\mathrm{L}^{\downarrow} \lambda$ out in the spectral regions outside the absorption feature were parameterized as the $1 \mathrm{~nm}$ mean value centered on the maximum radiance values, which were measured between 750 and $755 \mathrm{~nm}$ and between 772 and $777 \mathrm{~nm}$ in the $\mathrm{O}_{2}-\mathrm{A}$ band, and between 680 and $688 \mathrm{~nm}$ for the $\mathrm{O}_{2}-\mathrm{B}$ band. When the 3FLD is applied, the measurement made at the single reference wavelength ( $\lambda$ out) used in the SFLD is replaced by the average value measured at the two wavelengths outside of the absorption line ( $\mathrm{L}^{\uparrow} \lambda$ out and $\mathrm{L}^{\downarrow} \lambda$ out).

The Kruskall-Wallis test was performed to statistically test differences in the SIF retrieval using different spectroradiometers. When this test indicated significant results $(p \leqslant 0.05)$, the Wilcoxon-Mann-Whitney test was employed to determine which spectroradiometer had a significantly different SIF retrieval $(p \leqslant 0.05)$, as compared to the others.

\subsection{Leaf Level Fluorescence Emission Measurements}

Leaf fluorescence emissions were also measured on ten grass leaves collected from the target and immediately (10 $\mathrm{min}$ ) analyzed in the laboratory. Leaf fluorescence emission spectra were measured over the wavelength range from 600 to $810 \mathrm{~nm}$ with a spectrofluorometer (Fluorolog-3, Model FL3-22, Spex Industries, Edison, NJ, USA). A monochromatic excitation wavelength set to $530 \mathrm{~nm}$ was used to simulate solar radiation, activating fluorescence. Fluorescence emissions were then measured between $640 \mathrm{~nm}$ and $810 \mathrm{~nm}$ at a sampling resolution of $1 \mathrm{~nm}$. The spectrofluorometer was calibrated with NIST traceable silicon trap detectors and provides simulated SIF intensities reported in $\mathrm{mW} \cdot \mathrm{m}^{-2} \cdot \mathrm{nm}^{-1} \cdot \mathrm{sr}^{-1}$.

The leaf level measurements collected cannot be directly compared with the measured top of canopy spectra, since the laboratory full emission spectrum measurements were induced by a monochromatic light, whereas at canopy scale, fluorescence was induced by sun and only retrieved at the $\mathrm{O}_{2}-\mathrm{B}$ and $\mathrm{O}_{2}-\mathrm{A}$ bands. TOC SIF estimates can be compared with other measurements obtained by active techniques, although, at current stage, the best way to evaluate the performance of the estimate relies on the use of canopy radiative transfer models (e.g., Soil Canopy Observation, Photochemistry and Energy fluxes (SCOPE), van der Tol et al. [34]). Overall, there is no formula for establishing the real magnitude of top of canopy SIF. Therefore the results of the experiment have been analyzed and discussed considering a priori knowledge derived from the literature (i.e., experimental estimates and SIF repeated behaviors) and comparing the obtained estimates with leaf level values. The estimates are considered acceptable once they are aligned with literature published values and agree with leaf level comparison.

\section{Results and Discussion}

\subsection{Depth of the Oxygen Absorption Bands}

The solar radiance spectra collected by the different spectroradiometers are shown in Figure 2. The graph reports the mean values of the 60 solar radiance spectra measured with the different spectroradiometers in the spectral range $(670-800 \mathrm{~nm})$ covered by all of the spectrometers. 

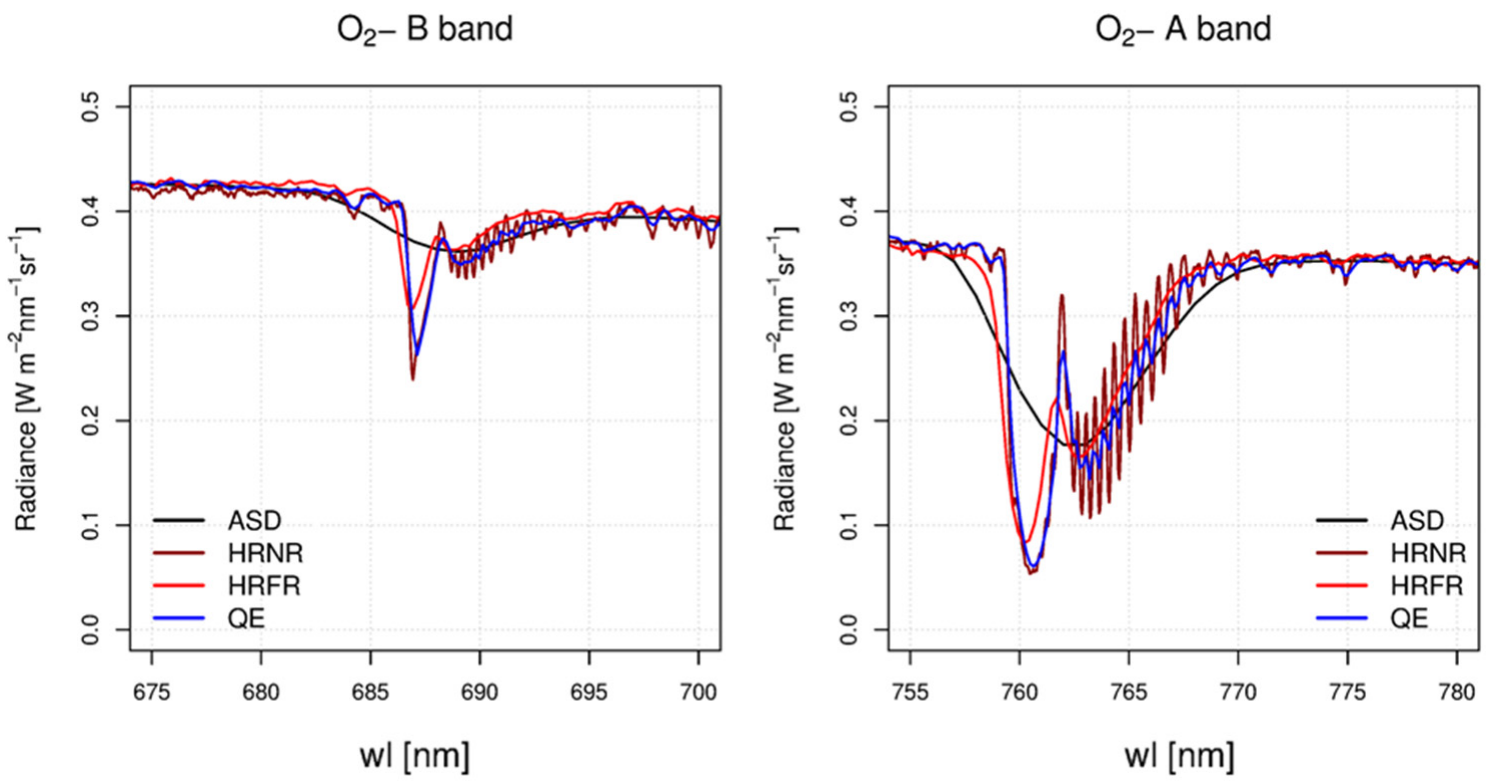

Figure 2. Incoming radiance measured with the four spectroradiometers in the $\mathrm{O}_{2}$ - $\mathrm{B}$ band (left) and in the $\mathrm{O}_{2}-\mathrm{A}$ band (right). Each color represents a different instrument. The ability to detect the presence of narrow absorption features is directly related to the Spectral Resolution (i.e., FWHM) of each device.

The spectra depicted in Figure 2 show comparable radiance values in the plateau spectral regions. However, due to their finer FWHM, a higher channel-to-channel variability occurred in the Ocean Optics spectroradiometers, as compared with the ASD spectroradiometer. Differences in FWHM among the instruments are also responsible for differences in describing the shape and depth of the two absorption bands (Table 1). The percent $\mathrm{O}_{2}-\mathrm{B}$ band depth estimates were lowest when made with the ASD (12\%), and highest (42\%) when made with the HRNR. Comparable but higher values were obtained for the $\mathrm{O}_{2}$-A band with the ASD (49\%) and the HRNR (81\%).

Table 1. The characteristics of each spectrometer are reported in terms of spectral range, spectral resolution (FWHM), spectral sampling interval (SSI), signal to noise ratio (SNR) and percentage depth of the two telluric oxygen absorption bands.

\begin{tabular}{ccccccc}
\hline Spectroradiometer & $\begin{array}{c}\text { Spectral } \\
\text { Range }[\mathbf{n m}]\end{array}$ & $\begin{array}{c}\text { FWHM } \\
{[\mathbf{n m}]}\end{array}$ & $\begin{array}{c}\text { SSI } \\
{[\mathbf{n m}]}\end{array}$ & $\begin{array}{c}\text { SNR } \\
{[-]}\end{array}$ & $\begin{array}{c}\mathbf{O}_{2} \text {-B Depth } \\
{[\%]}\end{array}$ & $\begin{array}{c}\mathbf{O}_{2} \text {-A Depth } \\
{[\%]}\end{array}$ \\
\hline$H R N R$ & $670-857$ & 0.2 & 0.05 & 250 & 42 & 81 \\
$Q E$ & $645-810$ & 0.5 & 0.17 & 1080 & 35 & 80 \\
$H R F R$ & $197-1115$ & 1 & 0.3 & 590 & 27 & 77 \\
$A S D$ & $350-2500$ & 5.5 & 1 & 1780 & 12 & 49 \\
\hline
\end{tabular}

Note that the shape of the incident radiance spectrum acquired with the HRNR (Figure 2, dark red line) is characterized by narrow absorption features, which are possible to discern with this instrument's higher spectral resolution, with FWHM of $0.2 \mathrm{~nm}$. These narrow features progressively disappear and the spectra appear to be progressively more smoothed as the spectral resolution of the spectroradiometers decreases for the QE, HRFR and ASD, as compared to the HRNR.

Moreover, an inverse relationship between the FWHM and the estimated depths of the telluric oxygen bands is apparent, with the feature depth decreasing from that acquired with the HRNR (with the smallest FWHM, $0.2 \mathrm{~nm}$ ) down to the lowest estimate acquired with the ASD (with the widest FWHM at $5.5 \mathrm{~nm}$ ) (Table 1). The percentage value of the $\mathrm{O}_{2}$ absorption band depths is known to vary according to environmental factors, such as the sun's zenith position the direct/diffuse shortwave radiation, and surface pressure. Therefore, the values presented in this manuscript are valid for this 
sensor intercomparison, under the specific conditions of the study and cannot be directly extrapolated to different geographic areas and atmospheric conditions.

\subsection{Performances of Different Portable Field Spectroradiometers on Fluorescence Estimates}

Figure 3 shows the fluorescence values retrieved from the spectroradiometers considered in the study: red (left panel) and far-red (right panel). The average retrievals per band and instrument are included in Table 2. At the far-red band, the QE and the two HR4000 (HRNR, HRFR) spectroradiometers retrieved the same SIF values (from Wilcoxon-Mann-Whitney test, $p$-value $\leqslant 0.05$ ). On the contrary, the ASD estimated a far-red SIF value that was six times higher than the mean value estimated using either HRNR, QE or HRFR. Estimates of the red SIF varied considerably among the different sensors, with values increasing in concert with the FWHM per sensor, with the lowest values obtained with the HRNR.
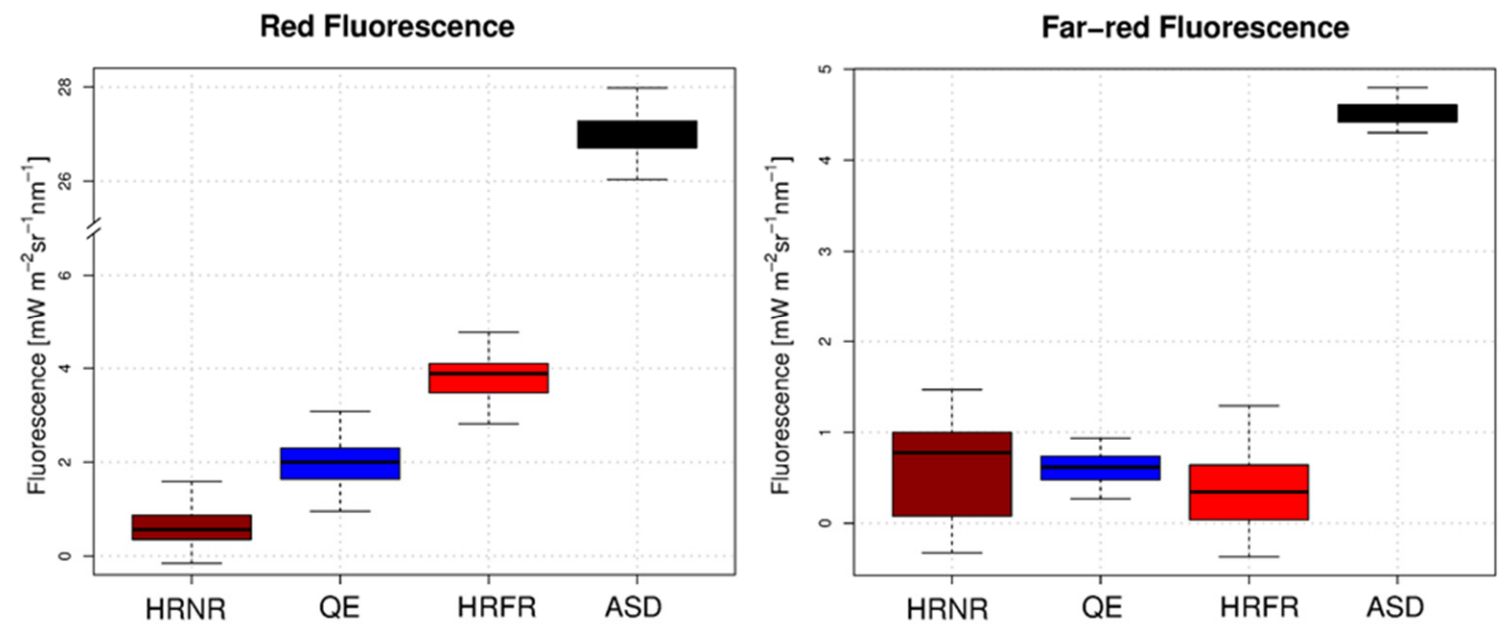

Figure 3. Boxplots of red (left) and far-red (right) Sun Induced chlorophyll Fluorescence (SIF) retrieval means and associated standard deviations of the 30 cycles of measurements. The instruments are reported in the order according to the increasing Full Width at Half Maximum (FWHM) and Spectral Sampling Interval (SSI). The red SIF estimations differ, indicating a possible dependency on the Spectral Resolution (SR) and SSI of the sensor. The HR4000 Narrow Range (HRNR), QE Pro and HR4000 Full Range show comparable far-red SIF retrieval estimates. SIF estimated from the ASD Fieldspec Pro is extremely high in both the red and far-red cases. The difference in the standard deviations for the far-red SIF estimates (right) can be explained by the differences in the Signal to Noise Ratio (SNR) of these instruments.

It is well known that different retrieval methods and technical device characteristics affect the SIF estimates $[20,22,23,35]$. Overall, in recent literature, few studies published the measured or modeled absolute TOC SIF values [12,36-40]. From the literature, the far-red SIF estimated at $760 \mathrm{~nm}$ is expected to range between 0.5 and $3 \mathrm{~mW} \cdot \mathrm{m}^{-2} \cdot \mathrm{sr}^{-1} \cdot \mathrm{nm}^{-1}$ according to different canopies. Far-red SIF values higher than $3 \mathrm{~mW} \cdot \mathrm{m}^{-2} \mathrm{sr}^{-1} \cdot \mathrm{nm}^{-1}$ have only been reported for a DCMU treated grassland [41]. Thus, a value higher than $3 \mathrm{~mW} \cdot \mathrm{m}^{-2} \cdot \mathrm{sr}^{-1} \cdot \mathrm{nm}^{-1}$ can be considered unrealistic for natural grassland. According to our results, we can affirm that absolute values of far-red SIF in line with literature values can be achieved using any of the higher resolution spectroradiometers examined (HRNR, QE and HRFR) with an average value of $0.7 \mathrm{~mW} \cdot \mathrm{m}^{-2} \cdot \mathrm{sr}^{-1} \cdot \mathrm{nm}^{-1}$, but not with the ASD. The Ocean Optics devices have an ultrafine resolution (FWHM $\leqslant 1 \mathrm{~nm}$ ) and a corresponding percentage depth of the $\mathrm{O}_{2}$-A band greater than $75 \%$, a value that, in our experiment, we should consider as a reasonable threshold to obtain reliable far-red SIF data. On the contrary, only a few studies report the absolute values of the red TOC SIF $[9,24,41-44]$. In all cases, the red SIF never exceeded the value of $3 \mathrm{~mW} \cdot \mathrm{m}^{-2} \cdot \mathrm{sr}^{-1} \cdot \mathrm{nm}^{-1}$. Contrary to the results obtained for the far-red SIF, there was no agreement among the four sensors examined 
for the red SIF. The red SIF retrieval exhibits a strong dependency of the absolute mean SIF values on the SR of the devices. This can be noticed even in the spectroradiometers having an ultrafine spectral resolution. SR and the SSI affect the retrievals within this narrow $\mathrm{O}_{2}-\mathrm{B}$ feature $(<5 \mathrm{~nm})$, in contrast to the retrieval for the wider $(\sim 8 \mathrm{~nm}) \mathrm{O}_{2}$-A NIR band. Figure 3 suggests that the estimated value of red SIF generally increases with the FWHM and the SSI of the spectroradiometer used for the retrieval. Thus, different configurations produce different results (Table 2). Based on these considerations, we are confident that only the estimates obtained by HRNR and QE give acceptable absolute values.

Red SIF values obtained with different spectroradiometers have a similar standard deviation (SD) while the SD varied among spectroradiometers for the far-red SIF estimation. We hypothesize that the differences in the SD of the far-red SIF is related to the SNR of the spectroradiometers [22], since the ASD and QE sensors had similarly low SDs. On the contrary, the higher radiance values recorded in correspondence of the $\mathrm{O}_{2}-\mathrm{B}$ band (at $687 \mathrm{~nm}$ ) may reduce the SNR impact, producing a lower SD variability within the red SIF estimates. For the far-red SIF, the smaller SDs for the QE and ASD sensors is related to their very high SNR (>1000). In addition, for the ASD, the small SD for the far-red SIF is related as well to the high number of scans averaged (25). ASD's large SIF overestimates in both red and far-red are due to the relatively low spectral resolution of the system (actual FWHM of $5.5 \mathrm{~nm}$ ). Further investigations are necessary to understand the capabilities of ASD to capture the relative variability of far-red SIF on targets emitting fluorescence with known intensities. Although instruments with high SNR value (SNR > 1000) are preferred for such measurements, it does not exclude the use of devices with a low SNR, but this would result in less reliable results. This implies a larger number of replicates needed to obtain less variable (i.e., more precise) mean values (e.g., with the HRNR and HRFR sensors). For example, it would be a simple revision in the data collection protocol for the HRNR to increase the number of scans utilized in order to lower the observed SD of measurements.

Overall, the results of the experiment suggest that spectroradiometers with a high spectral resolution (FWHM $\leqslant 1 \mathrm{~nm}$ ) are able to estimate the absolute value of far-red SIF, based on the 3FLD retrieval method. The $\mathrm{O}_{2}-\mathrm{B}$ absorption band is itself less wide compared to the $\mathrm{O}_{2}-\mathrm{A}$ band, introducing a physical constraint in the red SIF absolute value retrieval. Only the HRNR and QE device, which have the highest ultrafine spectral resolution gave the most reliable red SIF estimates, and are best retrieved using the sFLD method.

SIF estimates at the canopy level have been qualitatively compared with the leaf level fluorescence emissions at the same wavelengths. Figure 4 shows the leaf level fluorescence emissions acquired in the laboratory.

The spectrum obtained from leaves with 532-nm excitation form a valid estimate for the fluorescence spectrum, although it is not exactly the same as the sunlight-induced spectrum. In particular, absolute values of leaf and canopy fluorescence are expected to differ for two main reasons. First, the light source used to induce laboratory leaf emissions has a peak at $532 \mathrm{~nm}$, which is close to the wavelength peak of solar radiance, but the shape and intensity of the monochromatic light source differs from the natural solar irradiance. Moreover, current studies clearly indicate that the red fluorescence is generally reabsorbed by photosynthetic pigments, which are expected to influence the shape of the emission spectrum within the leaf [19] Furthermore, leaf fluorescence spectral properties are expected to be different at canopy level due to the variable and selective reabsorption of fluorescence by chlorophyll through the canopy [45]. 
Leaf level Fluorescence emission

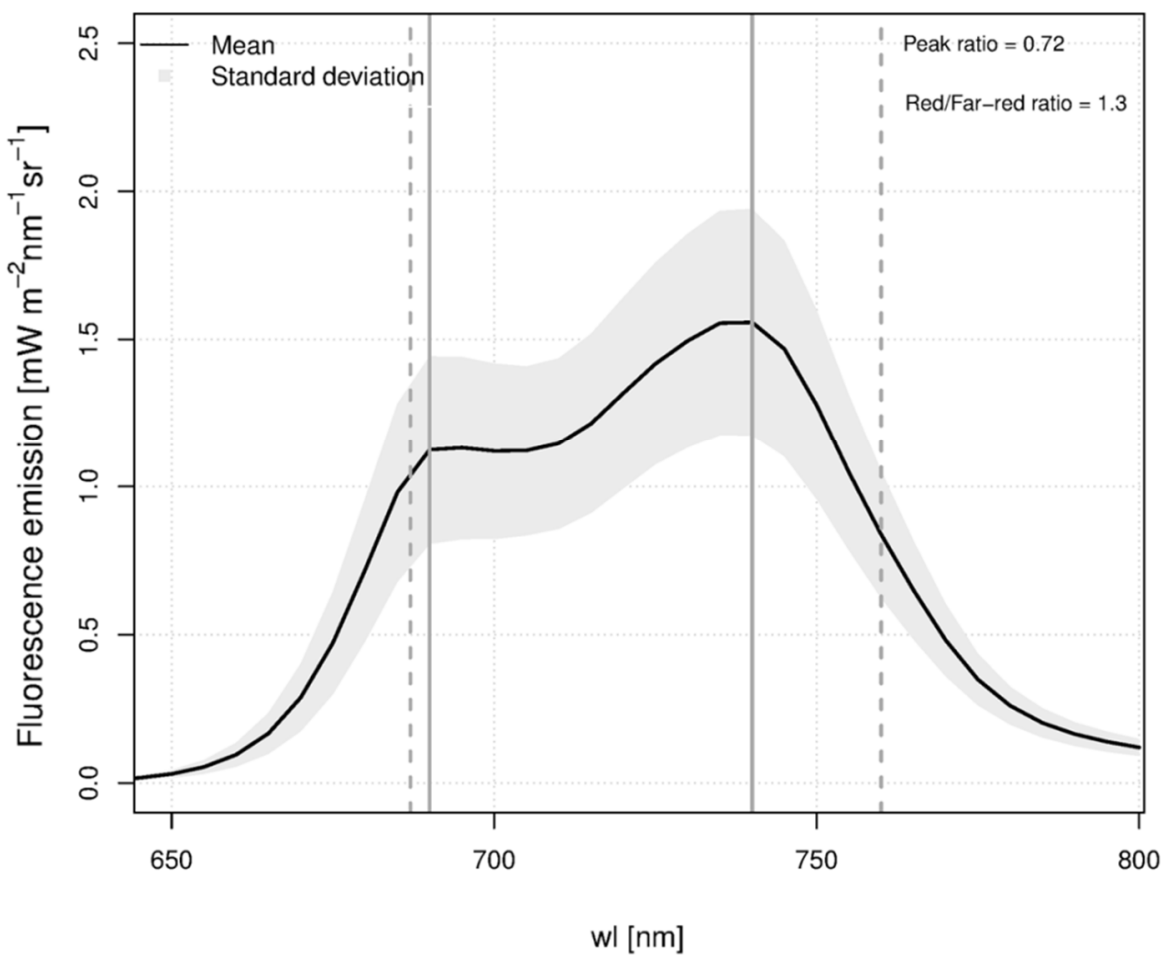

Figure 4. Grass leaf level fluorescence emissions acquired in the laboratory with the SPEX Fluorolog III spectrofluorometer using a $532 \mathrm{~nm}$ excitation wavelength. The red peak is expected to be partially reabsorbed at the canopy level. The ratio between fluorescence obtained at $689 \mathrm{~nm}$ and 760 (red/far-red ratio $=1.3$ ) is used to qualitatively assess the SIF retrievals made at canopy level with the different instruments (see Table 2). Traditionally, the red/far-red ratio is computed with the values at the peaks $(690 \mathrm{~nm}, 740 \mathrm{~nm})$, which would provide a far-red $=0.72$. The two solid vertical lines denote the peaks of the red and far-red fluorescence, whereas the dotted lines refer to the wavelengths used for the SIF estimation.

We computed the red/far-red SIF ratios (Table 2) and compared each with the leaf level value, to determine which spectrometer provided a ratio closest to the single leaf value. The measured leaf level red/far-red ratio value using the SIF wavelengths $(689 \mathrm{~nm}, 760 \mathrm{~nm})$ was 1.3 , whereas the traditional red/far-red peak ratio value using the peak wavelengths $(690 \mathrm{~nm}, 740 \mathrm{~nm})$ yields a value of 0.72 . HRNR and QE give a ratio which is close to that estimated from leaf level measurements, while HRFR and ASD can be considered out of range. HRNR is the instrument that provides the ratio closest to the leaf level value. However, considering the large variability of the red/far-red standard deviation, we cannot exclude that QE can also likely be used to retrieve absolute values of red SIF.

Table 2. The mean values and standard deviations of the red, far-red SIF estimates and red/far-red ratio are reported according to each spectrometer used.

\begin{tabular}{|c|c|c|c|}
\hline Spectroradiometer & $\begin{array}{l}\text { red Fluorescence Mean Value } \\
{\left[\mathbf{m W} \cdot \mathbf{m}^{-2} \cdot \mathbf{n} \mathbf{m}^{-1} \cdot \mathbf{s r}^{-1}\right]}\end{array}$ & $\begin{array}{l}\text { rar-red Fluorescence Mean } \\
\text { Value }\left[\mathbf{m W} \cdot \mathbf{m}^{-2} \cdot \mathbf{n m}^{-1} \cdot \mathbf{s r}^{-1}\right]\end{array}$ & $\begin{array}{l}\text { Red/Far-Red } \\
\text { Ratio [-] }\end{array}$ \\
\hline HRNR & $0.56 \pm 0.40$ & $0.77 \pm 0.50$ & $0.72 \pm 1.2$ \\
\hline$Q E$ & $2 \pm 0.48$ & $0.62 \pm 0.24$ & $3.2 \pm 1.5$ \\
\hline$\tilde{H R F R}$ & $3.8 \pm 0.42$ & $0.35 \pm 0.36$ & $10.9 \pm 4.2$ \\
\hline$A S D$ & $26.74 \pm 0.55$ & $4.52 \pm 0.14$ & $5.9 \pm 0.2$ \\
\hline
\end{tabular}


In summary, the TOC SIF results indicate that two of the four instruments examined, namely the HRNR and QE, have the highest potential for accurate field SIF measurements in both SIF bands. The HRFR gives reliable estimates for far-red SIF only, while the ASD spectroradiometer always overestimates the SIF values in both bands.

\section{Conclusions}

This study shows an empirical inter-comparison between spectroradiometers with different performance characteristics and their impacts on SIF retrievals. It should be considered a first step in evaluating the best instruments and measurement protocols for their use in field research and for measurements to validate space-based remote sensing retrievals. Our results indicate that different instruments provide different estimates with SNR and SR playing an important role. Regarding the red SIF, an ultra-fine spectral resolution is mandatory, while, for the far-red SIF estimates, the ultra-fine SR plays a minor role and different instruments can be used to achieve a reliable absolute value. Moreover, a SNR greater than 1000 is recommended for determining accurate SIF values. Nevertheless, the reliability of SIF estimates might be enhanced by adjusting the measurement protocol, using either more repeated scans or longer integration times. This study indicates that spectroradiometer configurations like those of the HRNR and QE can be successfully used for near-field quantitative measurements of TOC SIF, while the use of spectroradiometers configured with SR > $1 \mathrm{~nm}$ and SNR levels $<500$ are discouraged for FLD based SIF retrievals in the telluric $\mathrm{O}_{2}$ features.

Further study should address different topics that have only been partially investigated here. Different approaches in SIF retrieval (e.g., Spectral Fitting Method and statistical approaches) should be considered as it is well known that the performance of these algorithms may significantly impact the SIF estimates. New studies must be addressed to determine the optimal measurement configurations to boost the measurement success of HRNR and QE like spectroradiometers. Additional analyses are also needed to identify the SR limit in capturing relative changes of red SIF on vegetation targets with different fluorescence emissions. The results presented only refer to the particular instruments used, to the specific grass target considered, and to the methodologies used both in acquiring the measurements and in retrieving the SIF values. Nevertheless, credible results have been achieved, demonstrating the relative suitability for SIF retrieval of the instruments examined, and the potential impact on the reliability of in situ SIF values reported in the literature that were collected with similar field instruments as examined here.

Acknowledgments: This research was conducted in the framework of the FLEX-US Project, an ESA/NASA Joint Campaign for the Deployment of the Airborne HyPlant Imaging Spectrometer (ESA Contract No.4000109199/13/NL/FF/lf), which was co-sponsored by ESA and NASA. The authors are thankful to the COST actions ES1309 OPTIMISE and ES0903 EUROSPEC for enhancing scientific exchange on practical hyperspectral measurements. We also acknowledge Karolina Sakowska for drawing Figure 1.

Author Contributions: Tommaso Julitta designed the experiment with Micol Rossini. He collected the spectral data together with Lawrence A. Corp and Milton Hom, and analyzed the data. He wrote large parts of the manuscript. Elizabeth M. Middleton and Roberto Colombo supervised the analysis, defining the structure of the manuscript. Andreas Burkart, Sergio Cogliati, Anke Schickling, Alasdair Mac Arthur, Neville Davies and Uwe Rascher contributed to the manuscript by writing, editing, performing the layout and proofreading.

Conflicts of Interest: The authors declare no conflict of interest.

\section{References}

1. Maxwell, K.; Johnson, G.N. Chlorophyll fluorescence-A practical guide. J. Exp. Bot. 2000, 51, 659-668. [CrossRef] [PubMed]

2. Porcar-Castell, A.; Tyystjärvi, E.; Atherton, J.; van der Tol, C.; Flexas, J.; Pfündel, E.E.; Moreno, J.; Frankenberg, C.; Berry, J.A. Linking chlorophyll a fluorescence to photosynthesis for remote sensing applications: Mechanisms and challenges. J. Exp. Bot. 2014, 65. [CrossRef] [PubMed]

3. Campbell, P.K. E.; Middleton, E.M.; McMurtrey, J.E.; Corp, L.A.; Chappelle, E.W. Assessment of vegetation stress using reflectance or fluorescence measurements. J. Environ. Qual. 2007, 36. [CrossRef] [PubMed] 
4. Damm, A.; Elbers, J.; Erler, A.; Gioli, B.; Hamdi, K.; Hutjes, R.; Kosvancova, M.; Meroni, M.; Miglietta, F.; Moersch, A.; et al. Remote sensing of sun-induced fluorescence to improve modeling of diurnal courses of gross primary production (GPP). Glob. Chang. Biol. 2010, 16, 171-186. [CrossRef]

5. Panigada, C.; Rossini, M.; Meroni, M.; Cilia, C.; Busetto, L.; Amaducci, S.; Boschetti, M.; Cogliati, S.; Picchi, V.; Pinto, F.; et al. Fluorescence, PRI and canopy temperature for water stress detection in cereal crops. Int. J. Appl. Earth Obs. Geoinf. 2014, 30, 167-178. [CrossRef]

6. Meroni, M.; Rossini, M.; Guanter, L.; Alonso, L.; Rascher, U.; Colombo, R.; Moreno, J. Remote sensing of solar-induced chlorophyll fluorescence: Review of methods and applications. Remote Sens. Environ. 2009, 113, 2037-2051. [CrossRef]

7. Frankenberg, C.; O’Dell, C.; Berry, J.; Guanter, L.; Joiner, J.; Köhler, P.; Pollock, R.; Taylor, T.E. Prospects for chlorophyll fluorescence remote sensing from the Orbiting Carbon Observatory-2. Remote Sens. Environ. 2014, 147, 1-12. [CrossRef]

8. Hand, E. Remote sensing. Carbon-mapping satellite will monitor plants' faint glow. Science 2014, 344, 1211-1012. [CrossRef] [PubMed]

9. Cogliati, S.; Verhoef, W.; Kraft, S.; Sabater, N.; Alonso, L.; Vicent, J.; Moreno, J.; Drusch, M.; Colombo, R. Retrieval of sun-induced fluorescence using advanced spectral fitting methods. Remote Sens. Environ. 2015, 169, 344-357. [CrossRef]

10. Rossini, M.; Meroni, M.; Migliavacca, M.; Manca, G.; Cogliati, S.; Busetto, L.; Picchi, V.; Cescatti, A.; Seufert, G.; Colombo, R. High resolution field spectroscopy measurements for estimating gross ecosystem production in a rice field. Agric. For. Meteorol. 2010, 150, 1283-1296. [CrossRef]

11. Guanter, L.; Rossini, M.; Colombo, R.; Meroni, M.; Frankenberg, C.; Lee, J.-E.; Joiner, J. Using field spectroscopy to assess the potential of statistical approaches for the retrieval of sun-induced chlorophyll fluorescence from ground and space. Remote Sens. Environ. 2013, 133, 52-61. [CrossRef]

12. Daumard, F.; Champagne, S.; Fournier, A.; Goulas, Y.; Ounis, A.; Hanocq, J.-F.; Moya, I. A field platform for continuous measurement of canopy fluorescence. IEEE Trans. Geosci. Remote Sens. 2010, 48, 3358-3368. [CrossRef]

13. Guanter, L.; Zhang, Y.; Jung, M.; Joiner, J.; Voigt, M.; Berry, J.A.; Frankenberg, C.; Huete, A.R.; Zarco-Tejada, P.; Lee, J.-E.; et al. Global and time-resolved monitoring of crop photosynthesis with chlorophyll fluorescence. Proc. Natl. Acad. Sci. USA 2014, 111, E1327-E1333. [CrossRef] [PubMed]

14. Guanter, L.; Alonso, L.; Gomez-Chova, L.; Amorós-Lopez, J.; Vila, J.; Moreno, J. Estimation of solar-induced vegetation fluorescence from space measurements. Geophys. Res. Lett. 2007, 34. [CrossRef]

15. Joiner, J.; Yoshida, Y.; Vasilkov, A.P.; Corp, L.A.; Middleton, E.M. First observations of global and seasonal terrestrial chlorophyll fluorescence from space. Biogeosciences 2011, 8, 637-651. [CrossRef]

16. Joiner, J.; Yoshida, Y.; Vasilkov, A.P.; Middleton, E.M.; Campbell, P.K.E.; Kuze, A.; Corp, L.A. Filling-in of near-infrared solar lines by terrestrial fluorescence and other geophysical effects: Simulations and space-based observations from SCIAMACHY and GOSAT. Atmos. Meas. Tech. 2012, 5, 809-829. [CrossRef]

17. Joiner, J.; Guanter, L.; Lindstrot, R.; Voigt, M.; Vasilkov, A.P.; Middleton, E.M.; Huemmrich, K.F.; Yoshida, Y.; Frankenberg, C. Global monitoring of terrestrial chlorophyll fluorescence from moderate-spectral-resolution near-infrared satellite measurements: Methodology, simulations, and application to GOME-2. Atmos. Meas. Tech. 2013, 6, 2803-2823. [CrossRef]

18. Lee, J.-E.; Frankenberg, C.; van der Tol, C.; Berry, J.A.; Guanter, L.; Boyce, C.K.; Fisher, J.B.; Morrow, E.; Worden, J.R.; Asefi, S.; et al. Forest productivity and water stress in Amazonia: Observations from GOSAT chlorophyll fluorescence. Proc. Biol. Sci. 2013, 280, 20130171. [CrossRef] [PubMed]

19. Buschmann, C. Variability and application of the chlorophyll fluorescence emission ratio red/far-red of leaves. Photosynth. Res. 2007, 92, 261-271. [CrossRef] [PubMed]

20. Meroni, M.; Busetto, L.; Colombo, R.; Guanter, L.; Moreno, J.; Verhoef, W. Performance of spectral fitting methods for vegetation fluorescence quantification. Remote Sens. Environ. 2010, 114, 363-374. [CrossRef]

21. Plascyk, J.A. The MK II Fraunhofer Line Discriminator /FLD-II/ for airborne and orbital remote sensing of solar-stimulated luminescence. Opt. Eng. 1975, 14, 339-346. [CrossRef]

22. Damm, A.; Erler, A.; Hillen, W.; Meroni, M.; Schaepman, M.E.; Verhoef, W.; Rascher, U. Modeling the impact of spectral sensor configurations on the FLD retrieval accuracy of sun-induced chlorophyll fluorescence. Remote Sens. Environ. 2011, 115, 1882-1892. [CrossRef] 
23. Meroni, M.; Barducci, A.; Cogliati, S.; Castagnoli, F.; Rossini, M.; Busetto, L.; Migliavacca, M.; Cremonese, E.; Galvagno, M.; Colombo, R.; et al. The hyperspectral irradiometer, a new instrument for long-term and unattended field spectroscopy measurements. Rev. Sci. Instrum. 2011, 82, 043106. [CrossRef] [PubMed]

24. Fournier, A.; Daumard, F.; Champagne, S.; Ounis, A.; Goulas, Y.; Moya, I. Effect of canopy structure on sun-induced chlorophyll fluorescence. ISPRS J. Photogramm. Remote Sens. 2012, 68, 112-120. [CrossRef]

25. Drolet, G.; Wade, T.; Nichol, C.J.; MacLellan, C.; Levula, J.; Porcar-Castell, A.; Nikinmaa, E.; Vesala, T. A temperature-controlled spectrometer system for continuous and unattended measurements of canopy spectral radiance and reflectance. Int. J. Remote Sens. 2014, 35, 1769-1785. [CrossRef]

26. Louis, J.; Ounis, A.; Ducruet, J.-M.; Evain, S.; Laurila, T.; Thum, T.; Aurela, M.; Wingsle, G.; Alonso, L.; Pedros, R.; et al. Remote sensing of sunlight-induced chlorophyll fluorescence and reflectance of Scots pine in the boreal forest during spring recovery. Remote Sens. Environ. 2005, 96, 37-48. [CrossRef]

27. Cogliati, S.; Rossini, M.; Julitta, T.; Meroni, M.; Schickling, A.; Burkart, A.; Pinto, F.; Rascher, U.; Colombo, R. Continuous and long-term measurements of reflectance and sun-induced chlorophyll fluorescence by using novel automated field spectroscopy systems. Remote Sens. Environ. 2015, 164, 270-281. [CrossRef]

28. Porcar-Castell, A.; MacArthur, A.; Rossini, M.; Eklundh, L.; Pacheco-Labrador, J.; Anderson, K.; Balzarolo, M.; Martín, M.P.; Jin, H.; Tomelleri, E.; et al. EUROSPEC: At the interface between remote sensing and ecosystem $\mathrm{CO}_{2}$ flux measurements in Europe. Biogeosciences. 2015, 12, 6103-6124. [CrossRef]

29. Burkart, A.; Schickling, A.; Mateo, M.P.C.; Wrobel, T.J.; Rossini, M.; Cogliati, S.; Julitta, T.; Rascher, U. A Method for uncertainty assessment of passive sun-induced chlorophyll fluorescence retrieval using an infrared reference light. IEEE Sens. J. 2015, 15, 4603-4611. [CrossRef]

30. Schaepman, M.E.; Dangel, S. Solid laboratory calibration of a nonimaging spectroradiometer. Appl. Opt. 2000, 39, 3754. [CrossRef] [PubMed]

31. Busetto, L.; Meroni, M.; Crosta, G.F.; Guanter, L.; Colombo, R. SpecCal: Novel software for in-field spectral characterization of high-resolution spectrometers. Comput. Geosci. 2011, 37, 1685-1691. [CrossRef]

32. Meroni, M.; Busetto, L.; Guanter, L.; Cogliati, S.; Crosta, G.F.; Migliavacca, M.; Panigada, C.; Rossini, M.; Colombo, R. Characterization of fine resolution field spectrometers using solar Fraunhofer lines and atmospheric absorption features. Appl. Opt. 2010, 49, 2858-2571. [CrossRef] [PubMed]

33. Meroni, M.; Colombo, R. 3S: A novel program for field spectroscopy. Comput. Geosci. 2009, 35, 1491-1496. [CrossRef]

34. Van der Tol, C.; Verhoef, W.; Timmermans, J.; Verhoef, A.; Su, Z. An integrated model of soil-canopy spectral radiances, photosynthesis, fluorescence, temperature and energy balance. Biogeosciences 2009, 6, 3109-3129. [CrossRef]

35. Cogliati, S.; Colombo, R.; Rossini, M.; Meroni, M.; Julitta, T.; Panigada, C. Retrieval of vegetation fluorescence from ground based and airborne high resolution measurements. IGARSS 2012, 2012, 7129-7132.

36. Rascher, U.; Alonso, L.; Burkart, A.; Cilia, C.; Cogliati, S.; Colombo, R.; Damm, A.; Drusch, M.; Guanter, L.; Hanus, J.; et al. Sun-induced fluorescence-A new probe of photosynthesis: First maps from the imaging spectrometer HyPlant. Glob. Chang. Biol. 2015, 21, 4673-4684. [CrossRef] [PubMed]

37. Damm, A.; Guanter, L.; Paul-Limoges, E.; van der Tol, C.; Hueni, A.; Buchmann, N.; Eugster, W.; Ammann, C.; Schaepman, M.E. Far-red sun-induced chlorophyll fluorescence shows ecosystem-specific relationships to gross primary production: An assessment based on observational and modeling approaches. Remote Sens. Environ. 2015, 166, 91-105. [CrossRef]

38. Zhang, Y.; Guanter, L.; Berry, J.A.; Joiner, J.; van der Tol, C.; Huete, A.; Gitelson, A.; Voigt, M.; Köhler, P. Estimation of vegetation photosynthetic capacity from space-based measurements of chlorophyll fluorescence for terrestrial biosphere models. Glob. Chang. Biol. 2014, 20, 3727-3742. [CrossRef] [PubMed]

39. Middleton, E.M.; Corp, L.A.; Campbell, P.K.E. Comparison of measurements and FluorMOD simulations for solar-induced chlorophyll fluorescence and reflectance of a corn crop under nitrogen treatments. Int. J. Remote Sens. 2008, 29, 5193-5213. [CrossRef]

40. Rossini, M.; Alonso, L.; Cogliati, S.; Damm, A.; Guanter, L.; Julitta, T.; Meroni, M.; Moreno, J.; Panigada, C.; Pinto, F.; et al. Measuring sun-induced chlorophyll fluorescence: An evaluation and synthesis of existing field data. In Proceedings of the 5th International Workshop on Remote Sensing of Vegetation Fluorescence, Paris, France, 22-24 April 2014. 
41. Rossini, M.; Nedbal, L.; Guanter, L.; Ač, A.; Alonso, L.; Burkart, A.; Cogliati, S.; Colombo, R.; Damm, A.; Drusch, M.; et al. Red and far red Sun-induced chlorophyll fluorescence as a measure of plant photosynthesis. Geophys. Res. Lett. 2015, 42, 1632-1639. [CrossRef]

42. Liu, X.; Liu, L.; Zhang, S.; Zhou, X. New spectral fitting method for full-spectrum solar-induced chlorophyll fluorescence retrieval based on principal components analysis. Remote Sens. 2015, 7, 10626-10645. [CrossRef]

43. Cheng, Y.B.; Middleton, E.M.; Zhang, Q.; Huemmrich, K.F.; Campbell, P.K.E.; Corp, L.A.; Cook, B.D.; Kustas, W.P.; Daughtry, C.S. Integrating solar induced fluorescence and the photochemical reflectance index for estimating gross primary production in a cornfield. Remote Sens. 2013, 5, 6857-6879. [CrossRef]

44. Middleton, E.M.; Cheng, Y.-B.; Campbell, P.E.; Huemmrich, K.F.; Corp, L.A.; Bernardes, S.; Zhang, Q.; Landis, D.R.; Kustas, W.P.; Daughtry, C.S.T.; et al. Multi-angle hyperspectral observations using fluoresence and PRI to detect plant stress and productivity in a cornfield. In Proceedings of the 9th EARSeL SIG Imaging Spectroscopy Workshop, Luxembourg City, Luxembourg, 14-16 April 2015; pp. 27-39.

45. Cendrero-Mateo, M.P.; Moran, M.S.; Papuga, S.A.; Thorp, K.R.; Alonso, L.; Moreno, J.; Ponce-Campos, G.; Rascher, U.; Wang, G. Plant chlorophyll fluorescence: Active and passive measurements at canopy and leaf scales with different nitrogen treatments. J. Exp. Bot. 2016, 67, 275-286. [CrossRef] [PubMed]

(C) 2016 by the authors; licensee MDPI, Basel, Switzerland. This article is an open access article distributed under the terms and conditions of the Creative Commons by Attribution (CC-BY) license (http://creativecommons.org/licenses/by/4.0/). 